\title{
Effect of suppression of local distortion on the magnetic, electrical, and thermal transport properties of the Cr-substituted bilayer manganite $\mathrm{LaSr}_{2} \mathrm{Mn}_{2} \mathrm{O}_{7}$
}

\author{
M. Matsukawa, ${ }^{1, *}$ M. Chiba, ${ }^{1}$ E. Kikuchi, ${ }^{1}$ R. Suryanarayanan, ${ }^{2}$ M. Apostu, ${ }^{2}$ S. Nimori, ${ }^{3}$ K. Sugimoto, ${ }^{4}$ and N. Kobayashi ${ }^{5}$ \\ ${ }^{1}$ Department of Materials Science and Technology, Iwate University, Morioka 020-8551, Japan \\ ${ }^{2}$ Laboratoire de Physico-Chimie de L'Etat Solide, CNRS, UMR8648 Universite Paris-Sud, 91405 Orsay, France \\ ${ }^{3}$ National Institute for Materials Science, Tsukuba 305-0047, Japan \\ ${ }^{4} X$-Ray Research Laboratory, Rigaku Corporation, Tokyo 196-8666, Japan \\ ${ }^{5}$ Institute for Materials Research, Tohoku University, Sendai 980-8577, Japan
}

(Received 20 August 2004; revised manuscript received 14 September 2005; published 15 December 2005)

\begin{abstract}
We have investigated magnetic, electrical, and thermal transport properties (Seebeck effect and thermal conductivity) of $\mathrm{LaSr}_{2} \mathrm{Mn}_{2-y} \mathrm{Cr}_{y} \mathrm{O}_{7}$ polycrystalline samples $(y=0.1,0.2,0.4$, and 0.6$)$. $\mathrm{The}^{\mathrm{Cr}^{3+}}$ substitution for $\mathrm{Mn}^{3+}$ sites causes a removal of $d_{x^{2}-y^{2}}$ orbital of $e_{g}$ electron, resulting in a volume shrinkage of the lattice. Magnetic measurements reveal the appearance of a glassy behavior for $\mathrm{Cr}$-doped samples, accompanied by both a collapse of the A-type antiferromagnetic structure and the growth of ferromagnetic clusters. Cr-doping effect on electrical transport strongly enhances an insulating behavior over a wide range of temperatures, while it suppresses a local minimum of thermoelectric power at lower temperatures. For all polycrystalline samples with Cr substitution, the variable-range-hopping conduction model gives a reasonable fit to both resistivities and Seebeck coefficients. The phonon thermal conduction gradually rises with increasing $\mathrm{Cr}$ content, which is in contrast to a typical impurity effect on thermal conductivity. We attribute this to a suppression of local lattice distortion through the introduction of Jahn-Teller inactive ions of $\mathrm{Cr}^{3+}$.
\end{abstract}

DOI: $10.1103 /$ PhysRevB.72.224422

PACS number(s): 75.47.Lx, 75.50.Lk

\section{INTRODUCTION}

The discovery of colossal magnetoresistance (CMR) effect in doped manganites with perovskite structure has stimulated considerable interest for the understanding of their physical properties. ${ }^{1}$ Though the insulator-to-metal transition and its associated CMR are well explained on the basis of the double-exchange (DE) model, it is pointed out that the dynamic Jahn-Teller (JT) effect due to the strong electron-phonon interaction, plays a significant role in the appearance of CMR as well as the DE interaction. ${ }^{2,3}$ Furthermore, Dagotto et al. propose a phase separation model where the ferromagnetic (FM) metallic and antiferromagnetic (AFM) insulating clusters coexist and their model strongly supports recent experimental studies on the physics of manganites. ${ }^{4,5}$

In bilayer manganites $\mathrm{La}_{2-2 x} \mathrm{Sr}_{1+2 x} \mathrm{Mn}_{2} \mathrm{O}_{7}$, in which a $\mathrm{MnO}_{2}$ bilayer is alternatively stacked with a $(\mathrm{La}, \mathrm{Sr})_{2} \mathrm{O}_{2}$ blocking layer along the $c$ axis, the physical properties strongly depend on hole doping level $x .{ }^{6}$ In particular, neutron diffraction study on half-doped $\mathrm{LaSr}_{2} \mathrm{Mn}_{2} \mathrm{O}_{7}(x=0.5)$ has revealed the coexistence of the A-type antiferromagnetic phase and CE-type antiferromagnetic charge-ordered/orbitalordered $(\mathrm{CO} / \mathrm{OO})$ phase. $^{7}$ It is well known that the CE-type $\mathrm{CO} / \mathrm{OO}$ state in cubic manganites is unstable against $\mathrm{Cr}$ substitution for $\mathrm{Mn}$ site, and light $\mathrm{Cr}$ doping up to a few percent yields a drastic collapse of the $\mathrm{CO} / \mathrm{OO}$ phase, resulting in a FM metallic phase even in the absence of any applied magnetic field. ${ }^{8,9}$ While several reports on the effect of $\mathrm{Cr}$ substitution on the physical properties of the cubic manganites have appeared, very few reports have appeared on such studies in the case of bilayer manganites. ${ }^{10,11}$ Here, we give some comments on pressure effect on a two-dimensional network of $\mathrm{MnO}_{6}$ octahedra in bilayer manganites $\mathrm{La}_{1.2} \mathrm{Sr}_{1.8} \mathrm{Mn}_{2} \mathrm{O}_{7}$. Argyriou et al. ${ }^{12}$ reported that the $\mathrm{Mn}-\mathrm{O}(3)-\mathrm{Mn}$ bond angle is almost unchanged by application of pressure, indicating no tilting of the $\mathrm{MnO}_{6}$ octahedra in the $a b$ plane. Thus, it is possible to examine the internal and external pressure effect in bilayered manganites, varying the bond length of the $\mathrm{MnO}_{6}$ octahedra but keeping the bond angle almost $180^{\circ}$. In this paper, we report magnetic, electrical, and thermal transport properties of single-phase $\mathrm{LaSr}_{2} \mathrm{Mn}_{2-y} \mathrm{Cr}_{y} \mathrm{O}_{7}$ polycrystalline samples $(y=0.1,0.2,0.4$, and 0.6$)$. The $\mathrm{Cr}$ substitution for Mn sites causes a monotonic shrink of the $a(b)$ axis in contrast with a gradual elongation of the $c$ axis, accompanied by $d_{x^{2}-y^{2}}$ orbital deficiencies of $e_{g}$ electron, as listed in Table I. The $3 d$ electronic state of $\mathrm{Cr}^{3+}$ ion is taken as $t_{2 g}^{3} e_{g}^{0}$ (spin quantum number $S=3 / 2$ ), resulting in undistorted $\mathrm{CrO}_{6}$ octahedron sites free from a local Jahn-Teller effect. This finding is quite reasonable with a volume shrink-

TABLE I. The lattice parameters $a$ and $c$, A-type AFM transition temperature $T_{N}$, spin-glass-like transition temperature $T_{S G}$. The $T_{N}$ is determined from a local maximum at higher $T$ in $\mathrm{ZFC}$ data while $T_{S G}$ is defined from the prominent peak located at low $T$. The lattice parameters of single-crystalline $\mathrm{LaSr}_{2} \mathrm{Mn}_{2} \mathrm{O}_{7}$ are taken from Ref. 15 .

\begin{tabular}{ccccc}
\hline \hline Sample $y$ & $a(\AA)$ & $c(\AA)$ & $T_{N}(\mathrm{~K})$ & $T_{S G}(\mathrm{~K})$ \\
\hline 0 & 3.8790 & 19.996 & 210 & \\
0.1 & 3.8716 & 20.020 & 175 & \\
0.2 & 3.8660 & 20.030 & 130 & \\
0.4 & 3.8571 & 20.062 & & 38 \\
0.6 & 3.8562 & 20.094 & & 34.5 \\
\hline \hline
\end{tabular}


age observed due to $\mathrm{Cr}$ doping because a removal of $d_{x^{2}-y^{2}}$ orbital from $\mathrm{Mn}^{3+}$ sites easily causes a suppression of local lattice distortion, as discussed later. In the parent material $\mathrm{LaSr}_{2} \mathrm{Mn}_{2} \mathrm{O}_{7}$, a majority phase of the A-type AFM state coexists with a minority phase of CE-type AFM chargeordered/orbital-ordered state. ${ }^{7}$ We focus our attention on the Cr-doping effect on the A-type AFM majority phase because it is expected that the $\mathrm{CO} / \mathrm{OO}$ minority phase is strongly suppressed by $\mathrm{Cr}$ doping.

\section{EXPERIMENT}

Polycrystalline samples of $\operatorname{LaSr}_{2} \mathrm{Mn}_{2-y} \mathrm{Cr}_{y} \mathrm{O}_{7}(y=0.1,0.2$, 0.4 , and 0.6) were synthesized by solid-state reaction of $\mathrm{La}_{2} \mathrm{O}_{3}, \mathrm{SrCO}_{3}, \mathrm{MnO}_{2}$, and $\mathrm{Cr}_{2} \mathrm{O}_{3}$ powders with high purity. The oxygen concentration of typical samples with $y=0.2$ and 0.6 was determined using the infrared absorption method because the existence of the $\mathrm{Cr}$ ions may affect the valence estimation of the $\mathrm{Mn}$ ion made by the chemical analysis. The composition of cations was examined by inductively coupled plasma analysis. For the $y=0.2$ and 0.6 samples, we got $\mathrm{La}_{1.02} \mathrm{Sr}_{2.01} \mathrm{Mn}_{1.83} \mathrm{Cr}_{0.19} \mathrm{O}_{6.99}$ at $y=0.2$ and $\mathrm{La}_{1.01} \mathrm{Sr}_{1.95} \mathrm{Mn}_{1.41} \mathrm{Cr}_{0.59} \mathrm{O}_{7.05}$ at $y=0.6$. Thus, we conclude that our samples prepared by the solid-state reaction technique are close to nominal compositions. Let us consider the difference in oxygen concentration (hole concentration). The values of $7-\delta=6.99$ at $y=0.2$ and $7-\delta=7.05$ at $y=0.6$ give hole contents of $x=0.5$ and $x=0.55$, respectively. Recent neutron powder diffraction studies on $\mathrm{La}_{2-2 x} \mathrm{Sr}_{1+2 x} \mathrm{Mn}_{2} \mathrm{O}_{7}$ revealed the magnetic and crystallographic phase diagram in the region $x>0.5 .{ }^{13}$ In particular, when $0.42<x<0.66$, the A-type AFI state with antiferromagnetic coupling appears along the $c$ axis between FM single layers within one bilayer. We believe that the excess oxygen content $(x=0.55)$ has little effect on the magnetic property because the AFM magnetic transition temperature is stable over the range of hole concentration up to $x=0.6$.

The x-ray powder diffraction patterns were recorded for all samples on a RIGAKU diffractometer with $\mathrm{Cu} K \alpha$ radiation, as depicted in Fig. 1. The $\mathrm{x}$-ray data are indexed in terms of $(\mathrm{La}, \mathrm{Sr})_{3} \mathrm{Mn}_{2} \mathrm{O}_{7} 327$ phase except for a small amount of impurity phase, indicating a single phase of bilayered structure. The lattice parameters calculated using the least-squares fits are listed in Table I as a function of $\mathrm{Cr}$ content.

Magnetic measurements as a function of temperature were carried out using a superconducting quantum interference device magnetometer in both zero-field-cooled (ZFC) and field-cooled (FC) scans. The magnetic relaxation was measured as follows. First, the sample was cooled down to the respective temperatures in a zero field and then the applied field was held for $5 \mathrm{~min}$. Finally, just after the field was switched off, remanent magnetization data were recorded as a function of time. Electrical resistivity was measured by a conventional four-probe technique. Magnetoresistance measurements were performed at National Institute for Materials Science. Here, an electric current supplied was parallel to the direction of the external field. The thermal conductivity was measured using a conventional heat-flow method. The ther-
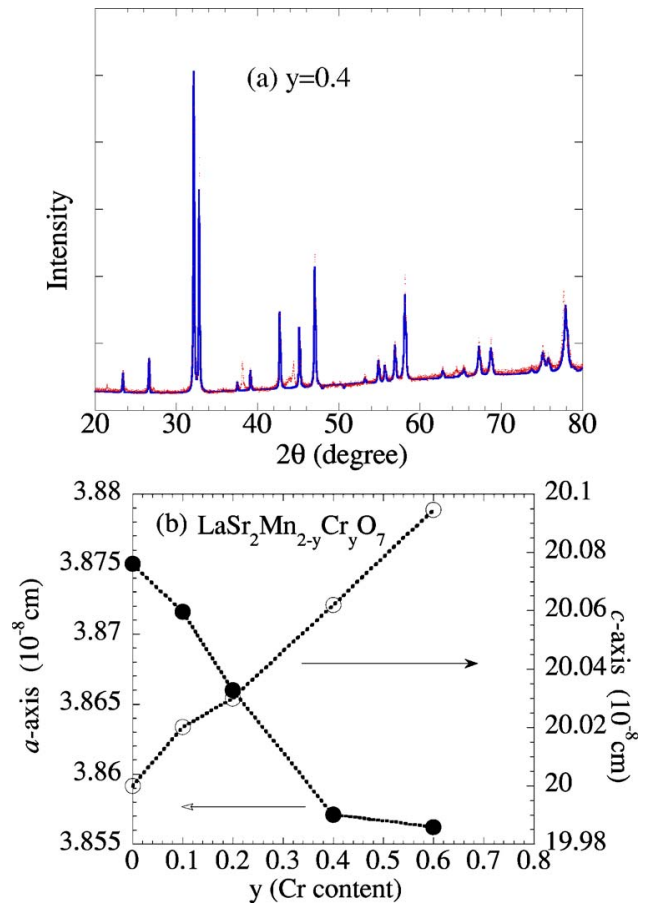

FIG. 1. (Color online) (a) The x-ray powder diffraction pattern on the $y=0.4$ sample. Dots and a solid line are the observed and calculated intensities, respectively. The x-ray data are indexed in terms of $(\mathrm{La}, \mathrm{Sr})_{3} \mathrm{Mn}_{2} \mathrm{O}_{7} 327$ phase except for a small amount of impurity phase. (b) The lattice parameters calculated using the least-squares fits as a function of $\mathrm{Cr}$ content, as listed in Table I.

moelectric power $S(=d V / d T)$ was determined both from a temperature gradient and thermoelectric voltage, $d T$ and $d V$, which are generated from a thermal current in the longitudinal direction of samples.

\section{RESULTS AND DISCUSSION}

\section{A. Magnetic property}

First, we show in Fig. 2 the ZFC and FC temperature dependences of the magnetization in polycrystalline $\mathrm{LaSr}_{2} \mathrm{Mn}_{2-y} \mathrm{Cr}_{y} \mathrm{O}_{7}(y=0.1,0.2,0.4$, and 0.6), measured at 10 $\mathrm{mT}$. For comparison, the $a b$-plane magnetization data of parent crystal $\mathrm{LaSr}_{2} \mathrm{Mn}_{2} \mathrm{O}_{7}$ are presented in the inset of Fig. $2 .{ }^{14}$ Upon cooling the $\mathrm{Cr}$-free sample, a broad maximum in $\mathbf{M}_{a b}$ is observed near about $210 \mathrm{~K}$, associated with the A-type AFM transition. ${ }^{15,16} \mathrm{Cr}$ doping strongly suppresses Néel temperature $T_{N}$, from $210 \mathrm{~K}$ at $y=0$, through $175 \mathrm{~K}$ at $y=0.1$, down to $130 \mathrm{~K}$ at $y=0.2$, and such a magnetic anomaly finally disappears for the $y=0.4$ and 0.6 samples. The $T_{N}$ is determined from a local maximum at higher temperatures in ZFC data. In the A-type AFM structure, FM spins lying in $a b$ plane of the respective $\mathrm{MnO}_{2}$ single layer are antiferromagnetically coupled along the $c$ axis within a $\mathrm{MnO}_{2}$ double layer. We expect that a partial substitution of $\mathrm{Cr}^{3+}$ for $\mathrm{Mn}^{3+}$ sites causes $d_{x^{2}-y^{2}}$ orbital deficiencies of $e_{g}$ electron and weakens an AFM coupling working between respective single layers, resulting in an observed drop of $T_{N}$. Instead, a low- $T$ peak in ZFC scan rapidly grows with $\mathrm{Cr}$ doping, ac- 


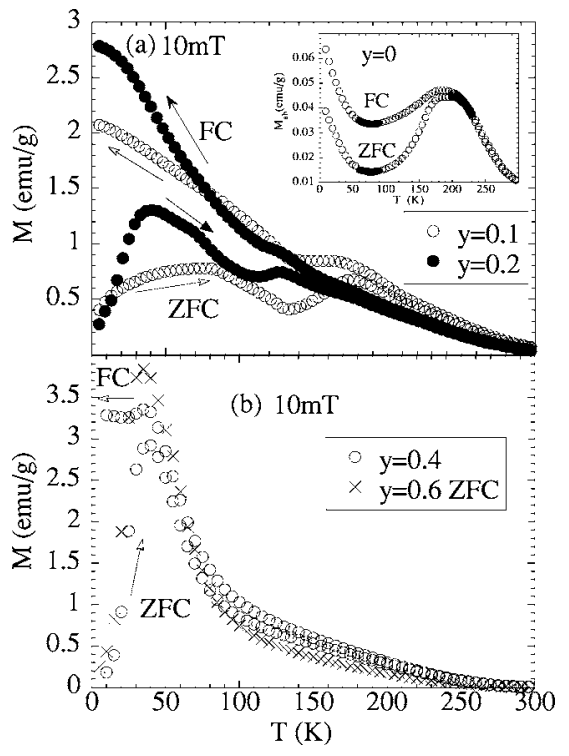

FIG. 2. ZFC and FC temperature dependences of the magnetization in polycrystalline $\mathrm{LaSr}_{2} \mathrm{Mn}_{2-y} \mathrm{Cr}_{y} \mathrm{O}_{7}(y=0.1,0.2,0.4$, and 0.6 ), measured at $10 \mathrm{mT}$. (a) $y=0.1$ and 0.2. (b) $y=0.4$ and 0.6. For comparison, the $a b$-plane magnetization data of parent crystal $\mathrm{LaSr}_{2} \mathrm{Mn}_{2} \mathrm{O}_{7}$ are presented in the inset of (a). The lower and upper data points (open circle) of (b) represent the ZFC and FC scan of the $y=0.4$ sample, respectively.

companied by a hysteresis region surrounded between ZFC and $\mathrm{FC}$ curves. At further low temperatures, the ZFC magnetization of $y=0.2-0.6$ shows a steep decrease, indicating the freezing of magnetic moments. ${ }^{17,18}$ These findings are reminiscent of magnetic behaviors of a standard spin-glass system due to a magnetic frustration between ferromagnetic and antiferromagnetic interactions. ${ }^{19}$ A characteristic temperature at which the prominent peak in ZFC scan is located at low $T$ is defined as $T_{S G}$ for the $y=0.4$ and 0.6 samples at $10 \mathrm{mT}$. In addition, the temperature variation of the magnetization in polycrystalline $\mathrm{LaSr}_{2} \mathrm{Mn}_{2-y} \mathrm{Cr}_{y} \mathrm{O}_{7}(y=0.1,0.2$, and 0.4 ) both in 0.1 and $1 \mathrm{~T}$ are shown in Fig. 3. At $0.1 \mathrm{~T}$, a history effect between ZFC and FC scans remains visible at lower $T$. However, at a relatively high field of $1 \mathrm{~T}$, the irreversibility in magnetization curves is strongly suppressed and a ferromagnetic-like behavior appears at low temperatures. These tendencies depending on the applied fields are never observed in a conventional spin-glass system.

Next, we examine the field dependence of low- $T$ magnetization in $\mathrm{LaSr}_{2} \mathrm{Mn}_{2-y} \mathrm{Cr}_{y} \mathrm{O}_{7}(y=0.1,0.2,0.4$, and 0.6) [Fig. 4(a)]. The $a b$-plane magnetization of the Cr-free crystal shows a linear dependence on the field, in association with an AFM spin canting induced by the external field. ${ }^{16}$ On the other hand, in Cr-doped samples, $M-H$ curves rise rapidly at low fields and then tend to saturate up to a maximum field of $5 \mathrm{~T}$, indicating the development of ferromagnetic states. Upon increasing $\mathrm{Cr}$ doping, the initial $M$ shows a steeper rise. We show in Fig. 4(b) the saturated magnetization at $5 \mathrm{~T}$ plotted as a function of $\mathrm{Cr}$ content. We notice that the saturated magnetic moment $M_{\text {sat }}$ is almost independent of $\mathrm{Cr}$ impurities, in strong contrast to the Cr-substitution effect on low-field magnetization in the inset of Fig. 4(b). It is true that

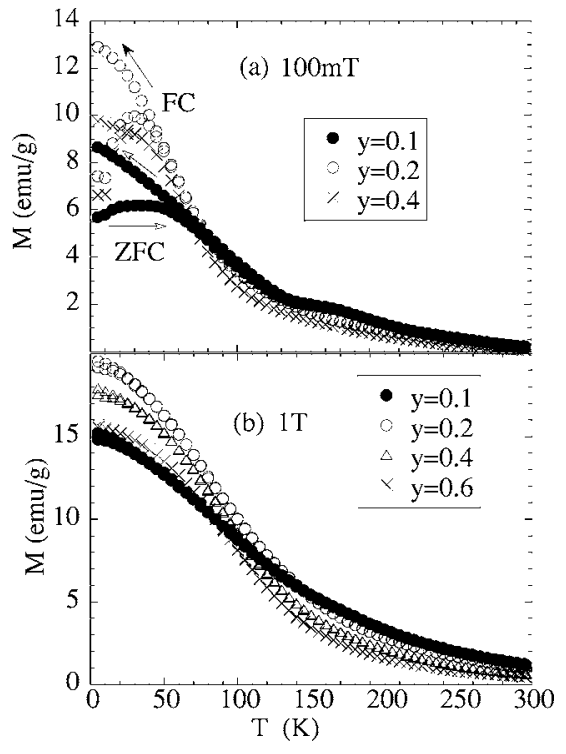

FIG. 3. FC and FC temperature dependences of the magnetization in polycrystalline $\mathrm{LaSr}_{2} \mathrm{Mn}_{2-y} \mathrm{Cr}_{y} \mathrm{O}_{7}(y=0.1,0.2$, and 0.4$)$, measured in fields of (a) $100 \mathrm{mT}$ and (b) $1 \mathrm{~T}$.

Cr impurity induces ferromagnetic moment from the inset of Fig. 4(b), but the volume fraction of FM phase at $5 \mathrm{~T}$ is almost insensitive of $\mathrm{Cr}$ content. The value of $M_{\text {sat }}(5 \mathrm{~T})$ is converged within $30 \%$ to $35 \%$ of full ferromagnetic moment. $\left(M_{\text {full }}=3.4 \mu_{B}\right.$ at $y=0.2$ and $M_{\text {full }}=3.2 \mu_{B}$ at $y=0.6$.) We give some comments on the apparent disagreement in the $\mathrm{Cr}$ -
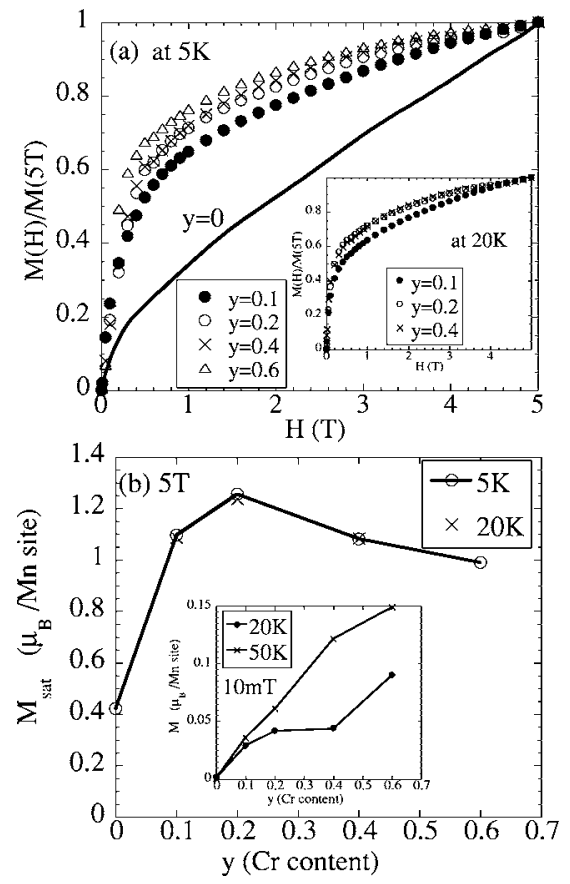

FIG. 4. (a) Field dependence of the magnetization at $5 \mathrm{~K}$ in $\mathrm{LaSr}_{2} \mathrm{Mn}_{2-y} \mathrm{Cr}_{y} \mathrm{O}_{7}(y=0.1,0.2,0.4$, and 0.6). The $M(H)$ data at 20 $\mathrm{K}$ are also given in the inset of (a). (b) The saturated magnetization at $5 \mathrm{~T}$ as a function of $\mathrm{Cr}$ content. For comparison, the low-field magnetization is plotted as a function of $\mathrm{Cr}$ content in the inset of (b) 

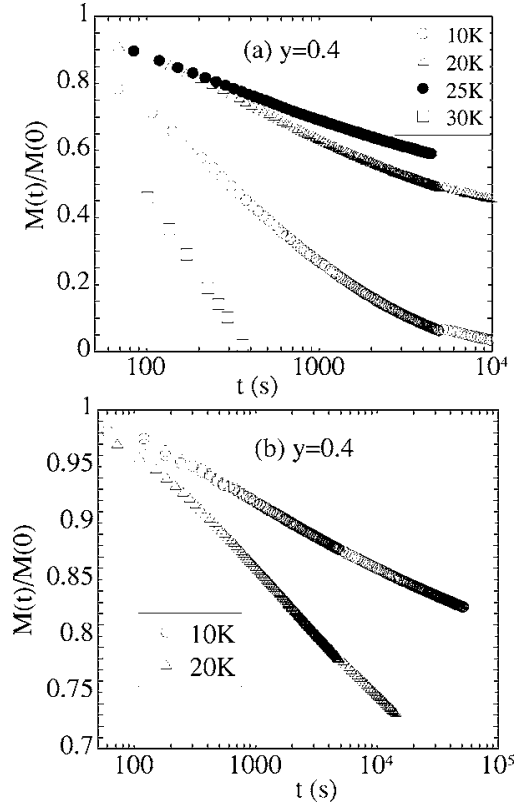

FIG. 5. Remanent magnetization data of the $y=0.4$ sample as a function of time, just after holding an applied field $H_{a}$ for 5 min and then switching it off. (a) $H_{a}=10 \mathrm{mT}$ and (b) $H_{a}=100 \mathrm{mT}$.

substitution effect between low- and high-field magnetic properties. A partial substitution of $\mathrm{Cr}^{3+}$ ion for $\mathrm{Mn}^{3+}$ suppresses not only AFM coupling between single $\mathrm{MnO}_{2}$ layers but also destroys FM double-exchange interaction between $\mathrm{Mn}^{3+}$ and $\mathrm{Mn}^{4+}$ ions within the $\mathrm{MnO}_{2}$ layer. It is expected that the addition of $\mathrm{Cr}^{3+}$ ions causes a suppression of the $\mathrm{FM}$ region mediated by $\mathrm{DE}$ interaction through removing $\mathrm{Mn}^{3+}$ ions. On the other hand, the low-field data support the occurrence of the ferromagnetic moment induced by $\mathrm{Cr}$ substitution. Following the Kanamori-Goodenough rules, the superexchange (SE) interaction between $\mathrm{Cr}^{3+}\left(t_{2 g}^{3} e_{g}^{0}\right)$ and $\mathrm{Mn}^{3+}$ $\left(t_{2 g}^{3} e_{g}^{1}\right)$ ions is ferromagnetic, while the SE interaction between $\mathrm{Cr}^{3+}$ and $\mathrm{Mn}^{4+}\left(t_{2 g}^{3} e_{g}^{0}\right)$ becomes antiferromagnetic. ${ }^{20}$ The annihilation of the $\mathrm{Mn}^{3+}-\mathrm{Mn}^{4+} \mathrm{FM}$ pairs is compensated by the creation of the $\mathrm{Cr}^{3+}-\mathrm{Mn}^{3+} \mathrm{FM}$ pairs accompanied by the $\mathrm{Cr}^{3+}-\mathrm{Mn}^{4+}$ AFM pairs. In other words, the DE-driven FM regions are partially replaced by the SE-driven FM regions with increasing the $\mathrm{Cr}$ ions, keeping the total FM fraction. The FM double-exchange interaction between $\mathrm{Mn}^{3+}$ and $\mathrm{Cr}^{3+}$ is not possible in our samples because the occurrence of FM moment by $\mathrm{Cr}$ doping accompanies no metallic property, as discussed later in the Cr-doping effect on resistivity. At high fields, the phase separation between the field-induced FM phase and AFM second phase is probably realized at the level of clusters on the basis of the competition between FM and AFM interaction.

Next, we carried out the magnetic relaxation of the $y=0.4$ sample in order to examine the glassy state below $T_{S G}$. In Fig. 5, we show the remanent magnetization data of the $y=0.4$ sample as a function of time, just after holding an applied field for $5 \mathrm{~min}$ and then switching it off. At $10 \mathrm{mT}$, the magnetization relaxes more quickly at lower $T$, in contrast with the $M(t)$ data at $100 \mathrm{mT}$. However, at $1 \mathrm{~T}$ no slow relaxation in $M$ is observed, which is consistent with no his-

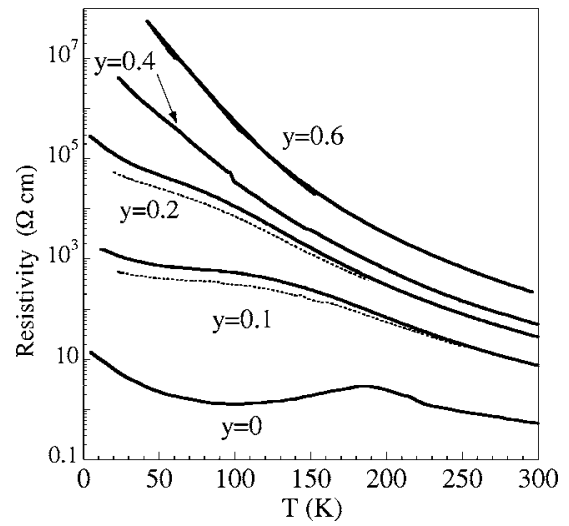

FIG. 6. Temperature dependence of the electrical resistivity in polycrystalline $\mathrm{LaSr}_{2} \mathrm{Mn}_{2-y} \mathrm{Cr}_{y} \mathrm{O}_{7}(y=0.1,0.2,0.4$, and 0.6) at zero field (solid lines) and an applied field of $8 \mathrm{~T}$ (dashed lines). For comparison, the $a b$-plane resistivity data of single crystalline $\mathrm{LaSr}_{2} \mathrm{Mn}_{2} \mathrm{O}_{7}$ are also presented.

tory effect in ZFC and FC scans. The slow decay of remanent magnetization curves indicates that a difference in free energy between the present excited and ground states is quite small in comparison with thermal energy, and the system remains stable in various excited states. ${ }^{21-23}$ Thus, a relatively fast relaxation of remanent $M$ at $10 \mathrm{~K}$ in the $10 \mathrm{mT}$ scan leads to a larger difference of energy barrier between the ground and excited states than in the case of $100 \mathrm{mT}$ at the same temperature. The metastable state excited by the lower field is probably related to the degree of a magnetic frustration between AFM and FM clusters and/or the spatial distribution of frustrated clusters. Furthermore, the coexistence of frustrated clusters and ferromagnetic clusters plays a crucial role in the magnetic relaxation in $100 \mathrm{mT}$. FM spins and/or FM domain walls are pinned on the lattice defect sites like an oxygen vacancy, giving a longer relaxation time.

\section{B. Electrical transport property}

Figure 6 displays the temperature dependence of the electrical resistivity in polycrystalline $\mathrm{LaSr}_{2} \mathrm{Mn}_{2-y} \mathrm{Cr}_{y} \mathrm{O}_{7}(y=0.1$, $0.2,0.4$, and 0.6). For comparison, the resistivity data of parent crystal $\mathrm{LaSr}_{2} \mathrm{Mn}_{2} \mathrm{O}_{7}$ are also presented in Fig. 6. The value of $\rho$ at lower $T$ exhibits a rapid increase by about four orders of magnitude, from $\sim 10^{2} \Omega \mathrm{cm}$ at $y=0.1$ up to $10^{6} \Omega \mathrm{cm}$ at $y=0.4$. Cr doping strongly enhances an insulating behavior over a wide range of temperatures because conduction paths are partially destroyed by $d_{x^{2}-y^{2}}$ orbital deficiencies of the $e_{g}$ electron. Our data exclude in this system a possibility of the global double-exchange interaction between $\mathrm{Mn}^{3+}$ and $\mathrm{Cr}^{3+}$ ions, giving a metallic property. ${ }^{24} \mathrm{In}$ particular, for the $y=0-0.2$ sample, the rapid rise in $\rho(T)$ below $50 \mathrm{~K}$ is close to a carrier localization effect due to a suppression of carrier hopping between single layers because at lower- $T$, orbital fluctuation of $d_{x^{2}-y^{2}}$ is gradually suppressed and motion of carriers is confined within the respective single layer. ${ }^{16}$

We try to analyze the $\rho(T)$ data of Cr-doped samples using the small-polaron hopping model and Mott's variable- 

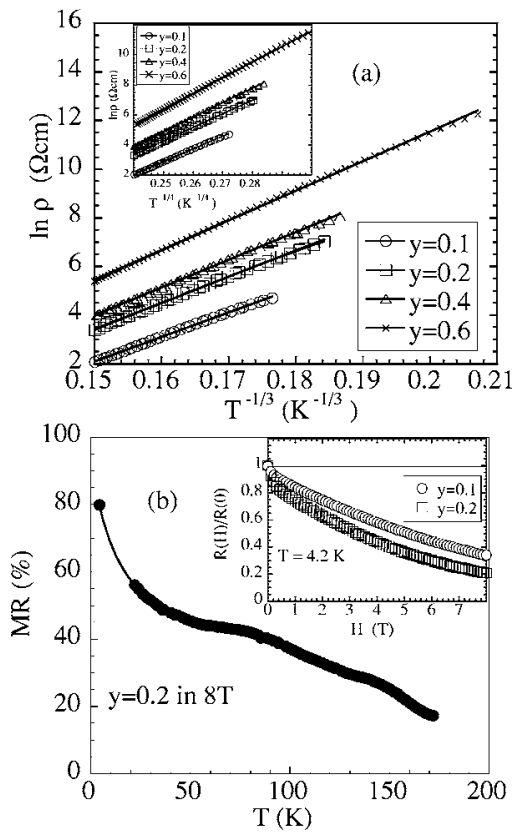

FIG. 7. (a) A semilog plot of $\rho$ versus $T^{-p}$ with $p=1 / 3$ for $\mathrm{LaSr}_{2} \mathrm{Mn}_{2-y} \mathrm{Cr}_{y} \mathrm{O}_{7}(y=0.1,0.2,0.4$, and 0.6). The solid lines correspond to fits by Mott's VRH model. The inset also presents a semi$\log$ plot of $\rho$ versus $T^{-p}$ with $p=1 / 4$ for 3D VRH. With increasing Cr-doping level, the VRH regime is extended, as listed in Table II. (b) Magnetoresistance effect of the $y=0.2$ sample as a function of temperature at $8 \mathrm{~T}$. In the inset, the MR of $y=0.1$ and 0.2 samples at $4.2 \mathrm{~K}$ is plotted as a function of field up to $8 \mathrm{~T}$.

range-hopping (VRH) model, ${ }^{25}$ to examine the conduction mechanism of bilayered manganites. ${ }^{26}$ According to Mott's VRH model, the temperature dependence of resistivities is represented by $\rho(T)=\rho_{0} \exp \left[\left(T_{0} / T\right)^{p}\right]$, where $\rho_{0}$ is a constant and $p=1 /(d+1)$, with $d$ being the dimensionality of the system. Mott's activation energy $T_{0}$ is proportional to $1 /\left[N(E) \xi^{d}\right]$, where $N(E)$ is the density of states at the Fermi level and $\xi$ is the localization length. On the other hand, the adiabatic small-polaron model is described by $\rho(T)=\rho_{0} T \exp \left(E_{\rho} / k T\right)$, where $\rho_{0}$ is a constant and $E_{\rho}$ represents the activation energy of a small polaron. For all samples with $\mathrm{Cr}$ substitution, it is found that the VRH model gives a more reasonable fit to the experimental data over a wide range of temperatures, in comparison with the smallpolaron model. In Fig. 7(a), we present our results as a semi$\log$ plot of $\rho$ versus $T^{-p}$ with $p=1 / 3$ for two-dimensional (2D) VRH, while the inset of Fig. 7(a) shows a semilog plot of $\rho$ versus $T^{-p}$ with $p=1 / 4$ for three-dimensional (3D) VRH. Although it is hard to distinguish a $T^{-1 / 3}$ or $T^{-1 / 4}$ dependence of $\ln \rho$, we obtain a much better fit to Mott's VRH than to a VRH model with $p=1 / 2$ in the presence of a Coulomb gap. ${ }^{26,27}$ The fitting parameters, $\rho_{0}$ and $T_{0}$, for polycrystalline samples of $\mathrm{LaSr}_{2} \mathrm{Mn}_{2-y} \mathrm{Cr}_{y} \mathrm{O}_{7}(y=0.1,0.2,0.4$, and 0.6) are listed in Table II. With increasing $\mathrm{Cr}$ content, the value of $T_{0}$ shows a monotonic increase for both $2 \mathrm{D}$ and $3 \mathrm{D}$ cases, indicating the decrease of the localization length $\xi$. The localization effect, enhanced due to $\mathrm{Cr}$ substitution, is probably associated with orbital disorders in $\mathrm{Mn}-\mathrm{O}-\mathrm{Mn}$ networks introduced by the removal of the $e_{g}$ electron. ${ }^{28,29}$
TABLE II. The fitting parameters $\rho_{0}$ and $T_{0}$ for polycrystalline samples of $\mathrm{LaSr}_{2} \mathrm{Mn}_{2-y} \mathrm{Cr}_{y} \mathrm{O}_{7}(y=0.1,0.2,0.4$, and 0.6).

\begin{tabular}{ccccccc}
\hline \hline & & \multicolumn{2}{c}{ 2D VRH } & & \multicolumn{2}{c}{$3 \mathrm{D}$ VRH } \\
\cline { 5 - 6 } $\begin{array}{c}\text { Sample } \\
y\end{array}$ & $\begin{array}{c}\text { VRH } \\
\text { regime }(\mathrm{K})\end{array}$ & $\rho_{0}(\Omega \mathrm{cm})$ & $T_{0}(\mathrm{~K})$ & $\rho_{0}(\Omega \mathrm{cm})$ & $T_{0}(\mathrm{~K})$ \\
\hline 0.1 & $T>187$ & $2.1 \times 10^{-6}$ & $1.0 \times 10^{6}$ & $8.7 \times 10^{-9}$ & $5.4 \times 10^{7}$ \\
0.2 & $T>161$ & $3.0 \times 10^{-6}$ & $1.2 \times 10^{6}$ & $8.1 \times 10^{-9}$ & $7.0 \times 10^{7}$ \\
0.4 & $T>155$ & $2.0 \times 10^{-6}$ & $1.5 \times 10^{6}$ & $3.7 \times 10^{-9}$ & $8.9 \times 10^{7}$ \\
0.6 & $T>113$ & $2.6 \times 10^{-6}$ & $1.8 \times 10^{6}$ & $2.7 \times 10^{-9}$ & $1.2 \times 10^{8}$ \\
\hline \hline
\end{tabular}

We give some comments on the doping effect of other trivalent metallic ions $\left(\mathrm{Co}^{3+}\right.$ and $\left.\mathrm{Al}^{3+}\right)$ on the $\mathrm{Mn}$ sites of $\mathrm{La}_{1} \mathrm{Sr}_{2} \mathrm{Mn}_{2} \mathrm{O}_{7}{ }^{28,29}$ The $3 d$ electronic configuration of $\mathrm{Co}^{3+}$ ion follows as $t_{2 g}^{6} e_{g}^{0} \quad\left(S=0\right.$, low-spin state), $t_{2 g}^{5} e_{g}^{1} \quad(S=1$, intermediate-spin state), and $t_{2 g}^{4} e_{g}^{2}(S=2$, high-spin state). The $\mathrm{Al}^{3+}$ ion is a nonmagnetic ion without $d$ electrons. With increasing $\mathrm{Co}^{3+}\left(\right.$ or $\mathrm{Al}^{3+}$ ) doping level, the A-type AFM temperature shifts to low temperatures and the magnitude of magnetization decreases over a wide range of temperatures. The decrease of $M$ implies a reduction of the net magnetic moments, which is consistent with low-spin state $(S=0)$ of $\mathrm{Co}^{3+}$ or nonmagnetic ion of $\mathrm{Al}^{3+}$. The latter tendency is in strong contrast with the magnetic effect of $\mathrm{Cr}^{3+}\left(t_{2 g}^{3} e_{g}^{0}, S=3 / 2\right)$ doping on $\mathrm{La}_{1} \mathrm{Sr}_{2} \mathrm{Mn}_{2} \mathrm{O}_{7}$, although a suppression of A-type AFM temperature is commonly observed for $\mathrm{Cr}, \mathrm{Co}$, and $\mathrm{Al}$ doping. On the other hand, the doping effects on electrical transport for $\mathrm{Cr}, \mathrm{Co}$, and $\mathrm{Al}$ ions exhibit such common features as the enhanced insulating state due to orbital deficiencies following the VRH model. In particular, the Al substitution without $d$ electrons for the Mn site produces a more rapid increase in resistivities.

Magnetoresistance effect of the $y=0.2$ sample as a function of temperature is depicted in Fig. 7(b), where the negative MR is defined as $-100[\rho(8 \mathrm{~T})-\rho(0 \mathrm{~T})] / \rho(0 \mathrm{~T})$. The value of giant MR increases from $25 \%$ at $150 \mathrm{~K}$ up to $80 \%$ at $4.2 \mathrm{~K}$ with decreasing $T$. The existence of the field-induced FM clusters is probably related to the enhanced MR at low temperatures, as we see from $M-T$ data in Fig. 3(b). In the inset of Fig. 7(b), the MR of $y=0.1$ and 0.2 samples is plotted as a function of field up to $8 \mathrm{~T}$. Cr doping also increases a low- $T$ MR from $45 \%$ at $y=0.2$ up to $80 \%$ at $y=0.6$ under a field of $8 \mathrm{~T}$ at $40 \mathrm{~K}$. The Cr-doping-induced orbital disorders assist charge transfer along the $c$ axis across the respective single layers of $\mathrm{MnO}_{2}$, giving the enhanced MR effect.

\section{Thermal transport properties (Seebeck coefficient and thermal conductivity)}

Next, the temperature variation of Seebeck coefficient $S$ for the $y=0.1-0.6$ samples is displayed in Fig. 8(a). For comparison, the $S(T)$ data of single crystalline $\mathrm{LaSr}_{2} \mathrm{Mn}_{2} \mathrm{O}_{7}$ are cited. ${ }^{14}$ For $y=0-0.2$, with decreasing $T$, the value of $S(T)$ shows a local maximum near the A-type AFM transition temperature $T_{N}$, and then a shallow minimum at lower $T$ is observed. ${ }^{30}$ At lower $T$, Cr doping gradually suppresses a local minimum of $S(T)$ from a negative value at $y=0$ down 

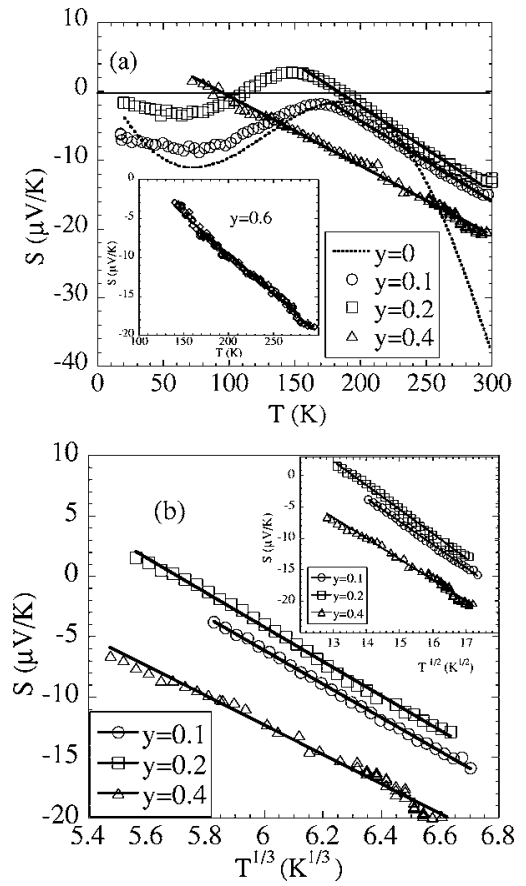

FIG. 8. (a) Temperature variation of Seebeck coefficient $S$ for $\mathrm{LaSr}_{2} \mathrm{Mn}_{2-y} \mathrm{Cr}_{y} \mathrm{O}_{7}(y=0,0.1,0.2$, and 0.4$)$. The solid lines correspond to $T$ linear fits. The inset represents the $S$ data of the $y=0.6$ sample with a linear fit. (b) Seebeck coefficient $S(T)$ versus $T^{1 / 3}$ for $\mathrm{LaSr}_{2} \mathrm{Mn}_{2-y} \mathrm{Cr}_{y} \mathrm{O}_{7}(y=0.1,0.2$, and 0.4$)$. The solid lines correspond to fits by the 2D VRH model. The inset presents $S(T)$ versus $T^{1 / 2}$ for the $3 \mathrm{D} V \mathrm{VRH}$ model. In the case of $y=0.2$, we have typical fitting parameters $A=77 \mu \mathrm{V} / \mathrm{K}$ and $B=14 \mu \mathrm{V} / \mathrm{K}^{4 / 3}$ for $2 \mathrm{D}$ $\mathrm{VRH}\left(A=52 \mu \mathrm{V} / \mathrm{K}\right.$ and $B=3.8 \mu \mathrm{V} / \mathrm{K}^{3 / 2}$ for $\left.3 \mathrm{D} \mathrm{VRH}\right)$, where $S(T)=A-B T^{p}$. The $S(T)$ of the $y=0.2$ follows the VRH law for $169 \mathrm{~K} \leqslant T \leqslant 300 \mathrm{~K}$.

to a small one at $y=0.2$, and finally at $y=0.4$ the local minimum in $S(T)$ disappears, giving a monotonic decrease over the observed temperature range. Now, let us try to analyze the $S(T)$ data of Cr-doped samples using the extended Mott's VRH model to Seebeck coefficients. ${ }^{25,31}$ For the 2D VRH case, the corresponding form is described by $S(T) \propto T^{p}$ with $p=1 / 3(p=1 / 2$ for the 3D VRH case). In Fig. 8(b), we present our results as a linear plot of $S(T)$ versus $T^{1 / 3}$ for $2 \mathrm{D}$ VRH (in the inset, $S(T)$ versus $T^{1 / 2}$ for 3D VRH). In a similar way, we obtain a much better fit of $S(T)$ data to the VRH law than to the thermally activated $T$ dependence. Here, the Seebeck coefficient for a thermally activated case is expressed as $S(T)=k / e\left(E_{S} / k T\right)+S_{\infty}$, where $E_{S}$ is a thermal activation energy and $S_{\infty}$ denotes Seebeck coefficient in the hightemperature limit. In addition, the obvious differences among the $T, T^{1 / 2}$, and $T^{1 / 3}$ dependences we do not notice within our fitting procedures. In the random hopping system, the $T$-linear dependence of $S(T)$ is presented theoretically by Culter and Mott. ${ }^{32}$ The $T$-linear dependence of $S(T)$ in the insulating state is probably related to a random distribution of localized electronic states around the Fermi level, as reported in Seebeck coefficient of $\mathrm{Li}_{1+x} \mathrm{Ti}_{2-x} \mathrm{O}_{4}$ oxides. ${ }^{33}$

In a doped bilayer manganite with hole content $x=0.4$, the high-temperature behavior of $S(T)$ is well explained on the basis of a model of Zener polarons, where a Zener polaron
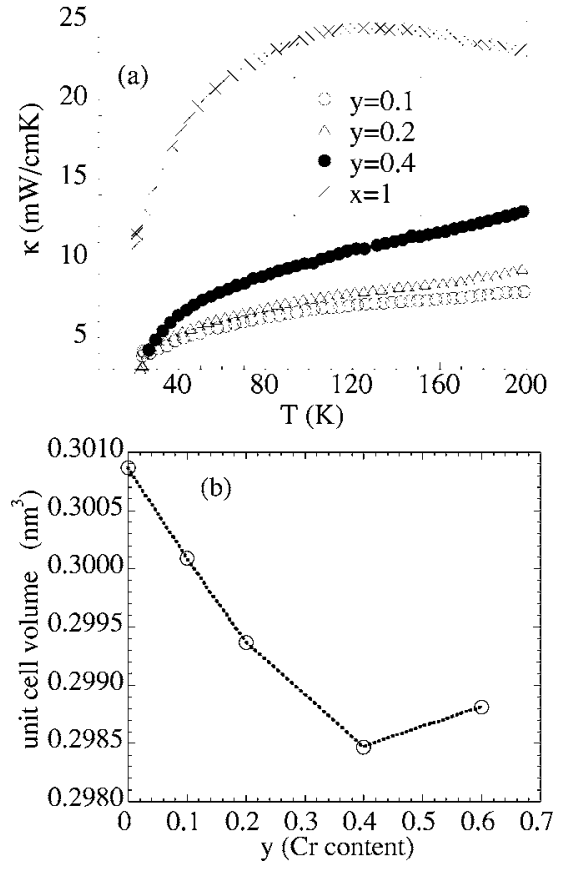

FIG. 9. (a) Temperature dependence of thermal conductivity for $\mathrm{LaSr}_{2} \mathrm{Mn}_{2-y} \mathrm{Cr}_{y} \mathrm{O}_{7}(y=0.1,0.2$, and 0.4). For comparison, the $\kappa$ data of polycrystalline $\mathrm{Sr}_{3} \mathrm{Mn}_{2} \mathrm{O}_{7}(x=1)$ are presented. (b) The unit-cell volume of $\mathrm{LaSr}_{2} \mathrm{Mn}_{2-y} \mathrm{Cr}_{y} \mathrm{O}_{7}$ as a function of $\mathrm{Cr}$ content.

formed in the high- $T$ region occupy two manganese sites. ${ }^{34}$ It is true that this model qualitatively reproduces a negative sign in high- $T$ behavior of single crystalline $\mathrm{La}_{1} \mathrm{Sr}_{2} \mathrm{Mn}_{2} \mathrm{O}_{7}$. However, for all polycrystalline samples with $\mathrm{Cr}$ substitution, it seems that the VRH conduction gives a reasonable fit to both resistivities and Seebeck coefficients.

Finally, let us show in Fig. 9 the thermal conductivity of Cr-doped $\mathrm{LaSr}_{2} \mathrm{Mn}_{2-y} \mathrm{Cr}_{y} \mathrm{O}_{7}(y=0.1,0.2$, and 0.4) as a function of temperature. For comparison, the $\kappa$ data of polycrystalline $\mathrm{Sr}_{3} \mathrm{Mn}_{2} \mathrm{O}_{7}(x=1)$ are presented. ${ }^{35}$ First of all, thermal carries are phonons since the electron component is estimated to be negligible from the resistivity data using the Weidemann-Franz law. The phonon thermal conduction gradually increases with $\mathrm{Cr}$ doping, which seems to be an unusual behavior because the introduction of Cr-impurity ions would disturb phonon conduction. However, this anomalous finding is reasonably resolved through clarifying a close relationship between phonon conduction and local lattice distortion of $\mathrm{MnO}_{6}$ due to the Jahn-Teller effect. In our previous work on thermal conductivity in bilayered manganite single crystals, it was made clear that the phonon conduction in the insulating state is scattered by local lattice distortions of $\mathrm{Mn}^{3+} \mathrm{O}_{6}$, but the metallic state realized by lowering of $T$ or by the applied field yields an upturn in $\kappa$ below $T_{C}$ or giant magnetothermal effect. ${ }^{35}$ This enhanced phonon conduction arises from a suppression of $\mathrm{Mn}^{3+} \mathrm{O}_{6}$ local distortions due to a screening effect of itinerent carriers. Cr substitution for $\mathrm{Mn}^{3+}$ sites removes $d_{x^{2}-y^{2}}$ orbitals of $e_{g}$ electron, resulting in a $\mathrm{Cr}^{3+} \mathrm{O}_{6}$ octahedron without a local JT effect. In other words, the Cr-doping effect on lattices causes a suppression of local lattice distortion through the introduction of JT inactive ions, giving an increase in phonon con- 
duction. Surely, the $\kappa(T)$ of polycrystalline $\mathrm{Sr}_{3} \mathrm{Mn}_{2} \mathrm{O}_{7}(x$ $=1$ ) shows a typical phonon conduction, whose behavior is free from JT distortion of $\mathrm{Mn}^{3+} \mathrm{O}_{6}$. In addition, the Crdoping dependence of $a$ - and $c$-axis lattice parameters in Table I reveals the volume shrinkage of the unit cell with increasing Cr content, as shown in Fig. 8(b). We note that the lattice constant of $y=0.6$ is influenced by a small amount of the impurity phase. This volume effect is associated with a number of deficiencies of $d_{x^{2}-y^{2}}$ orbitals of the $e_{g}$ electron, which is quite consistent with the preceding discussion on the close relationship between the lattice distortion and phonon conduction.

\section{SUMMARY}

We have carried out magnetic, electrical, Seebeck effect, and thermal conductivity measurements of $\mathrm{LaSr}_{2} \mathrm{Mn}_{2-y} \mathrm{Cr}_{y} \mathrm{O}_{7}$ polycrystalline samples $(y=0.1,0.2,0.4$, and 0.6$)$. The $\mathrm{Cr}^{3+}$ substitution for $\mathrm{Mn}^{3+}$ sites produces a monotonic shrink of the $a(b)$ axis in contrast with a gradual elongation of the $c$ axis in association with a removal of the $d_{x^{2}-y^{2}}$ orbital of the $e_{g}$ electron. For Cr-doped samples, a glassy behavior appears, accompanied by both a collapse of the A-type antiferromagnetic property and the development of ferromagnetic clusters. At high fields, the irreversibility in magnetization curves disappears and the saturated magnetic moment induced by the applied field reaches $30 \%$ to $35 \%$ of full ferro- magnetic moment at $5 \mathrm{~T}$ for all $\mathrm{Cr}$-doped samples. This finding strongly suggests the presence of a phase separation between FM and second phases at the level of clusters, which originates from the frustration between FM and AFM interactions. The electrical transport for $\mathrm{Cr}$-doped samples strongly enhances an insulating property over the wide range of temperatures because conduction paths are partially destroyed by $d_{x^{2}-y^{2}}$ orbital deficiencies of the $e_{g}$ electron. At lower $T, \mathrm{Cr}$ doping gradually suppresses a local minimum of $S(T)$ from a relatively large value at $y=0$ down to a positively small one at $y=0.4$, in striking contrast to the more enhanced low- $T$ resistivity data. For all polycrystalline samples with $\mathrm{Cr}$ substitution, it seems that the VRH conduction gives a reasonable fit to both resistivities and Seebeck coefficients. The phonon thermal conduction gradually increases with increasing $\mathrm{Cr}$ content, which is in contrast to a typical impurity effect on thermal conductivity. We propose that the increase in the phonon thermal conduction results from a suppression of local lattice distortion through the introduction of a Jahn-Teller inactive ion of $\mathrm{Cr}^{3+}$.

\section{ACKNOWLEDGMENTS}

This work was partially supported by a Grant-in-Aid for Scientific Research from the Ministry of Education, Science and Culture, Japan. The authors thank H. Noto and Dr. S. Ueda for their technical support.
*Electronic address: matsukawa@iwate-u.ac.jp

${ }^{1}$ Colossal Magnetoresistive Oxides, edited by Y. Tokura (Gordon and Breach, New York, 2000).

${ }^{2}$ C. Zener, Phys. Rev. 82, 403 (1951); P. G. deGennes, ibid. 118, 141 (1960).

${ }^{3}$ A. J. Millis, P. B. Littlewood, and B. I. Shraiman, Phys. Rev. Lett. 74, 5144 (1995); A. J. Millis, B. I. Shraiman, and R. Mueller, ibid. 77, 175 (1996).

${ }^{4}$ For a recent review, see E. Dagotto, T. Hotta, and A. Moreo, Phys. Rep. 344, 1 (2001).

${ }^{5}$ E. Dagotto, Nanoscale Phase Separation and Colossal Magnetoresistance (Springer-Verlag, Berlin, 2003).

${ }^{6}$ K. Hirota, Y. Moritomo, H. Fujioka, M. Kubota, H. Yoshizawa, and Y. Endoh, J. Phys. Soc. Jpn. 67, 3380 (1998).

${ }^{7}$ M. Kubota, H. Yoshizawa, Y. Moritomo, H. Fujioka, K. Hirota, and Y. Endoh, J. Phys. Soc. Jpn. 68, 2202 (1999).

${ }^{8}$ B. Raveau, A. Maignan, and C. Martin, J. Solid State Chem. 130, 162 (1997).

${ }^{9}$ T. Kimura, Y. Tomioka, R. Kumai, Y. Okimoto, and Y. Tokura, Phys. Rev. Lett. 83, 3940 (1999).

${ }^{10}$ R Gundakaram, J. L. Lin, F. Y. Lee, M. F. Tai, C. H. Shen, R. S. Liu, and C. H. Huang, J. Phys.: Condens. Matter 11, 5187 (1999).

${ }^{11}$ K. B. Chashka, B. Fisher, J. Genossar, A. Keren, L. Patlagan, G. M. Reisner, E. Shimshoni, and J. F. Mitchell, Phys. Rev. B 65, 134441 (2002).

${ }^{12}$ D. N. Argyriou, J. F. Mitchell, J. B. Goodenough, O. Chmaissem, S. Short, and J. D. Jorgensen, Phys. Rev. Lett. 78, 1568 (1997).
${ }^{13}$ C. D. Ling, J. E. Millburn, J. F. Mitchell, D. N. Argyriou, J. Linton, and H. N. Bordallo, Phys. Rev. B 62, 15096 (2000).

${ }^{14}$ M. Matsukawa, E. Kikuchi, M. Yoshizawa, M. Apostu, R. Suryanarayanan, A. Revcolevschi, and N. Kobayashi, Physica B 329333, 900 (2003).

${ }^{15}$ R. Suryanarayanan, G. Dhalenne, A. Revcolevschi, W. Prellier, J. P. Renard, C. Dupas, W. Caliebe, and T. Chatterji, Solid State Commun. 113, 267 (2000).

${ }^{16}$ T. Kimura, R. Kumai, Y. Tokura, J. Q. Li, and Y. Matsui, Phys. Rev. B 58, 11081 (1998).

${ }^{17}$ J. Dho, W. S. Kim, and N. H. Hur, Phys. Rev. B 65, 024404 (2002).

${ }^{18}$ M. Apostu, R. Suryanarayanan, A. Revcolevschi, H. Ogasawara, M. Matsukawa, M. Yoshizawa, and N. Kobayashi, Phys. Rev. B 64, 012407 (2001).

${ }^{19}$ J. A. Mydosh, Spin Glasses; an Experimental Introduction (Tayler \& Francis, London, 1993).

${ }^{20} \mathrm{~J}$. B. Goodenough, Magnetism and the Chemical Bond (Interscience, New York, 1963).

${ }^{21}$ R. Mathieu, P. Nordblad, D. N. H. Nam, N. X. Phuc, and N. V. Khiem, Phys. Rev. B 63, 174405 (2001).

${ }^{22}$ I. G. Deac, S. V. Diaz, B. G. Kim, S. W. Cheong, and P. Schiffer, Phys. Rev. B 65, 174426 (2002).

${ }^{23}$ I. Gordon, P. Wagner, V. V. Moshchalkov, Y. Bruynseraede, M. Apostu, R. Suryanarayanan, and A. Revcolevschi, Phys. Rev. B 64, 092408 (2001).

${ }^{24}$ Y. Sun, W. Tong, X. Xu, and Y. Zhang, Phys. Rev. B 63, 174438 (2001). 
${ }^{25}$ N. F. Mott and E. A. Davies, Electronic Processes in Noncrystalline Solids, 2nd ed. (Clarendon, Oxford, 1979).

${ }^{26}$ X. J. Chen, C. L. Zhang, J. S. Gardner, J. L. Sarrao, and C. C. Almasan, Phys. Rev. B 68, 064405 (2003).

${ }^{27}$ B. I. Shklovskii and A. L. Efros, Electronic Properties of Doped Semiconductors (Springer-Verlag, Berlin, 1984).

${ }^{28}$ R. L. Zhang, W. H. Song, Y. Q. Ma, J. Yang, B. C. Zhao, Z. G. Sheng, J. M. Dai, and Y. P. Sun, Phys. Rev. B 70, 224418 (2004).

${ }^{29}$ Sunil Nair and A. Banerjee, Phys. Rev. B 70, 104428 (2004).

${ }^{30} \mathrm{~S}$. Nakamae, D. Colson, A. Forget, I. Legros, J. F. Marucco, C.
Ayache, and M. Ocio, Phys. Rev. B 63, 092407 (2001).

${ }^{31}$ I. P. Zvyagin, in Hopping Transport in Solids, edited by M. Pollak and B. Shklowskii (North-Holland, Amsterdam, 1991).

${ }^{32}$ M. Culter and N. F. Mott, Phys. Rev. 181, 1336 (1969).

${ }^{33}$ I. Maekawa, F. Takagi, Y. Sakai, and N. Tsuda, J. Phys. Soc. Jpn. 56, 2119 (1987).

${ }^{34}$ J. S. Zhou, J. B. Goodenough, and J. F. Mitchell, Phys. Rev. B 58, R579 (1998).

${ }^{35}$ M. Matsukawa, M. Narita, T. Nishimura, M. Yoshizawa, M. Apostu, R. Suryanarayanan, A. Revcolevschi, K. Itoh, and N. Kobayashi, Phys. Rev. B 67, 104433 (2003). 